Egypt. Acad. J. Biolog. Sci., 5(1): 25-33 (2013)

G. Microbiology

Email:egyptianacademic@yahoo.com

ISSN: 2090-0872

Received: $20 / 3 / 2013$

www.eajbs.eg.net

\title{
Screening of some synthetic fused heterocyclic pyrimidines for anti-avian influenza virus (H5N1) activity
}

\author{
Omar R. Alfarouk ${ }^{1}$; Ahmed B. Barakat ${ }^{1}$;Sahar A. Shoman ${ }^{1}$; Husam A. Ghanem ${ }^{1}$ \\ ;Mohamed A. Ali ${ }^{2}$ and Aymn E. Rashad ${ }^{3}$ \\ 1 - Department of Microbiology, Faculty of Science, Ain Shams University, Cairo, Egypt. \\ 2- Department of water pollution, National Research Center, Dokki, Cairo, Egypt. \\ 3-Department of Photochemistry, National Research Center, Dokki,Cairo, Egypt.
}

\begin{abstract}
Thirteen chemical compounds belonging to the fused heterocyclic pyrimidines were synthesized and screened in order to assay their antiviral activity against the avian influenza virus (H5N1) A/Chicken/Qalubiya/1/2006(H5N1) by plaque reduction assay on MDCK cells. All tested compounds were non-toxic on the cells. Ten out of thirteen compounds $(10,13,9,12,1,11,7,8,3$ and 4$)$ showed moderate percentage of inhibition respectively at concentration $20 \mu \mathrm{g} / \mathrm{ml}$ while the remaining three compounds $(5,2$ and 6$)$ showed weak inhibitory activity respectively at same concentration.
\end{abstract}

Key words: Anti,avian influenza virus, H5N1 - pyrimidine derivatives.

\section{INTRODUCTION}

Avian influenza $\mathrm{A} / \mathrm{H} 5 \mathrm{~N} 1$ presents one of the major hazards to the mankind in the $21^{\text {st }}$ century. Influenza virus $\mathrm{A} / \mathrm{H} 5 \mathrm{~N} 1$ possesses a number of characteristics of the ideal future pandemic pathogen such as the following: the potential for the infection of the greatest number of animal species (compared to other known influenza strains), high mortality rate (30$70 \%$ ) as well as the continuous evolution towards the increase of virulence, the ability for human transmission and the resistance to the existing therapeutic agents. World Health Organization (WHO) has identified avian influenza $\mathrm{A} / \mathrm{H} 5 \mathrm{~N} 1$ as a disease with pandemic potential, which represents a health risk to the world population as a whole (Tapper, 2006). Periodically, completely novel antigenic subtypes of influenza viruses have been introduced in the human population, causing large-scale global outbreaks with high death tolls (Basler and Aguilar, 2008). Although vaccination provides the primary protection against influenza virus infections but the continuous and unpredictable antigenic variation in the influenza viruses has made vaccine strains relatively or totally ineffective beside they must be selected annually. Therefore, vaccine production may not satisfy the need during an influenza pandemic (Gong et al., 2007). The use of antiviral drugs against influenza virus could therefore represent a first line of defense against a new pandemic, allowing the control of the infection until sufficient quantities of a suitable vaccine can be produced (Sugrue et al., 2008). Two classes of these antiviral drugs, adamantanes and neuraminidase (NA) inhibitors, are currently prescribed for the prophylaxis and treatment of influenza infections (Hsieh and Hsu, 2007). Adamantanes (amantadine and rimantadine) target the proton channel formed by the viral M2 protein whereas two NA inhibitors, orally bioavailable oseltamivir and inhaled zanamivir, are the only drugs currently recommended for the 
treatment of influenza virus infection. These drugs have been proven to be useful for reducing clinical symptoms, but their utility has been limited by side effects and high rate of emergence of resistant viral strains (Gubareva et al., 1998; Kiso et al., 2004 and Le et al., 2005). Therefore the need for identifying new alternative antiinfluenza drugs becomes a matter of certainty and an urgent global need in the face of new pandemic strains. The objective of this study was to identify novel anti-influenza virus using heterocyclic pyrimidine compounds because an interest is aroused to the biologically active pyrimidine and fused heterocyclic pyrimidine nucleosides as potent antiviral agents (Cristescu and Czobor, 1998; Rashad and Ali, 2006).

\section{MATERIALS AND METHODS}

\section{I) Chemistry:}

A) Tested compounds: All chemical compounds under this investigation were kindly synthesized by Prof. Dr. Aymn Elsayed Rashad, Professor of Photochemistry, Photochemistry Department, National Research Centre and Prof. Dr. Klaus Banert Professor of Organic chemistry, Chemnitz University of Technology, Germany.

Compound number one: 1-(6-p-Tolylpyridazin-3-yl)-1, 5-dihydro-pyrazolo[3,4-d]pyrimidin-4-one was prepared by Shamroukh et al., (2005). M.p. 383-4 $4^{\circ}$ C. IR spectrum $\left(\mathrm{KBr}, v, \mathrm{~cm}^{-1}\right): 3102(\mathrm{NH})$, and $1700(\mathrm{CO}) ;{ }^{1} \mathrm{H}$ NMR spectrum (DMSO-d $6, \delta$ ppm): $2.41(\mathrm{~s}$, $3 \mathrm{H}, \mathrm{p}-\mathrm{CH}_{3}$-tolyl), $7.40\left(\mathrm{~d}, 2 \mathrm{H}, \mathrm{J}=7.8 \mathrm{~Hz}, \mathrm{H}_{3}\right.$, $\mathrm{H}_{5}$-tolyl), 8.11 (d, $2 \mathrm{H}, \mathrm{J}=7.5 \mathrm{~Hz}, \mathrm{H}_{2}, \mathrm{H}_{6}$ tolyl), 8.23 (s, $1 \mathrm{H}, \mathrm{H}_{3}$-pyrazole), $8.30(\mathrm{~d}, 1 \mathrm{H}, \mathrm{J}$ $=9.3 \mathrm{~Hz}, \mathrm{H}_{5}$-pyridazine $), 8.45-8.48(\mathrm{~m}, 2 \mathrm{H}$, $\mathrm{H}_{4}$-pyridazine and $\mathrm{H}_{6}$-pyrimidine) and 12.51 (brs, $1 \mathrm{H}, \mathrm{NH}$, exchangeable with $\mathrm{D}_{2} \mathrm{O}$ ). MS, $m / z \quad(\%): 304 \quad\left(\mathrm{M}^{+}, \quad 100\right)$. Analysis for $\mathrm{C}_{16} \mathrm{H}_{12} \mathrm{~N}_{6} \mathrm{O}$ (304.31): required $\mathrm{C}, 63.15 ; \mathrm{H}$, 3.97; N, 27.62; found $\mathrm{C}, 63.20 ; \mathrm{H}, 4.05 ; \mathrm{N}$, 27.48 .

Compound number two: 5-Amino-1-(6-ptolyl-pyridazin-3-yl)-1H-pyrazole-4-carboxylic acid amide was prepared by Shamroukh et al., (2005). M.p. $276-8^{\circ} \mathrm{C}$. IR spectrum $(\mathrm{KBr}, v$, $\left.\mathrm{cm}^{-1}\right)$ : 3423-3153 $\left(2 \mathrm{NH}_{2}\right)$, and $1662(\mathrm{CO}) ;{ }^{1} \mathrm{H}$ NMR spectrum (DMSO-d ${ }_{6}, \delta$ ppm): 2.40 (s, $3 \mathrm{H}, \quad$ p- $\mathrm{CH}_{3}$-tolyl), 7.10 (brs, $2 \mathrm{H}, \quad \mathrm{NH}_{2}$, exchangeable with $\left.\mathrm{D}_{2} \mathrm{O}\right), 7.39(\mathrm{~d}, 2 \mathrm{H}, \mathrm{J}=7.8$ $\mathrm{Hz}, \quad \mathrm{H}_{3}, \quad \mathrm{H}_{5}$-tolyl $), \quad 7.63 \quad\left(\mathrm{~s}, \quad 2 \mathrm{H}, \quad \mathrm{NH}_{2}\right.$, exchangeable with $\left.\mathrm{D}_{2} \mathrm{O}\right), 8.05-8.07(\mathrm{~m}, 3 \mathrm{H}$, $\mathrm{H}_{2}, \mathrm{H}_{6}$-tolyl and $\mathrm{H}_{3}$-pyrazole), $8.17(\mathrm{~d}, 1 \mathrm{H}, \mathrm{J}=$ $9.3 \mathrm{~Hz}, \mathrm{H}_{5}$-pyridazine $), 8.40(\mathrm{~d}, 1 \mathrm{H}, \mathrm{J}=9.3$ $\mathrm{Hz}, \mathrm{H}_{4}$-pyridazine); MS, m/z (\%): $294\left(\mathrm{M}^{+}\right.$, 100). Analysis for $\mathrm{C}_{15} \mathrm{H}_{14} \mathrm{~N}_{6} \mathrm{O}$ (294.32): required $\mathrm{C}, 61.22 ; \mathrm{H}, 4.79 ; \mathrm{N}, 28.55$; found $\mathrm{C}$, $61.12 ; \mathrm{H}, 4.58 ; \mathrm{N}, 28.75$.

Compound number three: 2Aminonaphtho[2,1-b]thiophene-1-carbonitrile was prepared by Rashad et al., (2010). M.p. 215-217 ${ }^{\circ} \mathrm{C}$. IR $\left(\mathrm{KBr}, \mathrm{n}, \mathrm{cm}^{-1}\right): 3390-3250$ $\left(\mathrm{NH}_{2}\right), 2218(\mathrm{C} \equiv \mathrm{N}) .{ }^{1} \mathrm{H}$ NMR $\left(\mathrm{DMSO}-\mathrm{d}_{6}, \delta\right.$ ppm): 5.29 (brs, $2 \mathrm{H}, \mathrm{NH}_{2}, \mathrm{D}_{2} \mathrm{O}$, exchangeable), 7.52-7.62 (m, 4H, Ar-H), $8.27(\mathrm{~d}, 1 \mathrm{H}, \mathrm{J}=4.8$ $\mathrm{Hz}, \mathrm{Ar}-\mathrm{H}), 8.99(\mathrm{~d}, 1 \mathrm{H}, \mathrm{J}=5.2 \mathrm{~Hz}, \mathrm{Ar}-\mathrm{H}) .{ }^{13} \mathrm{C}$ NMR (DMSO-d $\left.\mathrm{d}_{6}, \delta \mathrm{ppm}\right): 119.32(\mathrm{C} \equiv \mathrm{N}) 127-$ 145 ( $\mathrm{sp}^{2}$ carbon atoms). MS m/z (\%): $224\left(\mathrm{M}^{+}\right.$, 59\%). Anal. calcd. for $\mathrm{C}_{13} \mathrm{H}_{8} \mathrm{~N}_{2} \mathrm{~S}$ (224.29): $\mathrm{C}$ $69.62, \mathrm{H}$ 3.60, N 12.49. Found: C 69.49, H $3.51, \mathrm{~N} 21.64$.

Compound number four: 2-Aminoindeno [2,1-b]thiophene-3-carbonitrile was prepared by Hegab et al., (2007). IR; v 2280, 2200 $\left(\mathrm{NH}_{2}\right), 2210(\mathrm{CN}) .{ }^{1} \mathrm{H}$ NMR (DMSO-d $\mathrm{d}_{6}, \delta$ ppm): $3.75\left(\mathrm{~s}, 2 \mathrm{H}, \mathrm{CH}_{2}\right), 4.80\left(\mathrm{~s}, 2 \mathrm{H}, \mathrm{NH}_{2}\right.$, $\mathrm{D}_{2} \mathrm{O}$ exchangeable), 7.20-7.60 (m, 3H, Ar- $\left.\mathrm{H}\right)$, $7.75(\mathrm{~d}, \mathrm{~J}=6.00 \mathrm{~Hz}, 1 \mathrm{H}$, Ar-H). MS: $m / z 212$ $\left(\mathrm{M}^{+}, 100\right), 196$ (1.71), 184 (4.20).

Compound number five: 5,6,10Trihydronaphtho[1', $\left.\quad 2^{\prime}: 4,5\right]$ thieno[2,3d]pyrimidin-11-one was prepared by Rashad et al., (2005). M.p. $268-270^{\circ} \mathrm{C}$. Calcd. for $\mathrm{C}_{14} \mathrm{H}_{10} \mathrm{~N}_{2} \mathrm{OS}$ (254.31): C, 66.12; H, 3.96; N, 11.02; S, 12.61. Found: C, 66.20; H, 3.80; N, 10.83; S, 12.40. IR (KBr): $v_{\mathrm{NH}} \mathrm{cm}^{-1} 3100, v_{\mathrm{CO}}$ $1625 \mathrm{~cm}^{-1} .{ }^{1} \mathrm{H}$ NMR (DMSO-d 6 , $\delta \mathrm{ppm}$ ): $2.80-$ $3.10\left(\mathrm{~m}, 4 \mathrm{H}, 2 \mathrm{CH}_{2}\right), 7.20-7.50(\mathrm{~m}, 3 \mathrm{H}, \mathrm{Ar}-\mathrm{H})$, $8.10\left(\mathrm{~s}, 1 \mathrm{H}, \mathrm{C}_{9}-\mathrm{H}\right), 8.40(\mathrm{~d}, \mathrm{~J}=10.45 \mathrm{~Hz}, 1 \mathrm{H}$, Ar- $\mathrm{H}), 12.15$ (s, 1H, NH, $\mathrm{D}_{2} \mathrm{O}$ exchangeable). MS, $m / z$ (\%): $254\left(\mathrm{M}^{+}, 100\right), 226$ (5.67).

Compound number six: 11-(2-Methoxyethylsulfanyl) $\quad-5, \quad 6 \quad$-dihydronaphtho[1, $2: 4,5]$ thieno[2,3-d] pyrimidine was prepared by Rashad and Ali (2006). M.p. $102-104^{\circ} \mathrm{C}$; 
${ }^{1} \mathrm{H}-\mathrm{NMR}\left(\mathrm{CDCl}_{3}, \delta \mathrm{ppm}\right): 2.86-2.97(\mathrm{~m}, 4 \mathrm{H}$, $\left.2 \mathrm{CH}_{2}\right), 3.37\left(\mathrm{~s}, 3 \mathrm{H}, \mathrm{OCH}_{3}\right), 3.54(\mathrm{t}, \mathrm{J}=7 \mathrm{~Hz}, 2 \mathrm{H}$, $\left.\mathrm{CH}_{2} \mathrm{O}\right), 3.69\left(\mathrm{t}, \mathrm{J}=7.5 \mathrm{~Hz}, 2 \mathrm{H}, \mathrm{CH}_{2} \mathrm{~N}\right), 7.20$ $7.40(\mathrm{~m}, 3 \mathrm{H}, \mathrm{Ar}-\mathrm{H}), 7.80(\mathrm{~d}, \mathrm{~J}=8 \mathrm{~Hz}, 1 \mathrm{H}, \mathrm{Ar}-$ $\mathrm{H}), 8.25\left(\mathrm{~s}, 1 \mathrm{H}, \mathrm{C}_{9}-\mathrm{H}\right) ;{ }^{13} \mathrm{C}-\mathrm{NMR}\left(\mathrm{CDCl}_{3}, \delta\right.$ ppm): $25.56(\mathrm{C}-5), 29.78(\mathrm{C}-6), 29.78\left(\mathrm{OCH}_{3}\right)$, $58.89\left(\mathrm{CH}_{2} \mathrm{O}\right), 71.21\left(\mathrm{CH}_{2} \mathrm{~N}\right), 125.94-128.02$ (Ar-C), 130.97 (C-11a), 135.94 (C-11b), 141.24 (C-6a), 151.34 (C-7a), 162.98 (C-9), 166.22 (C-11); calculated for $\mathrm{C}_{17} \mathrm{H}_{16} \mathrm{~N}_{2} \mathrm{OS}_{2}$ (328.46): C, 62.17; H, 4.91; N, 8.53; S, 19.52. Found: C, 62.10; H, 4.85; N, 8.63; S, 19.60.

Compound number seven: 1,10 Dihydroindeno[ $\left[1^{\prime}, 2^{\prime}: 4, \quad 5\right]$ thieno[2,3d] $[1,2,4]$ triazolo[4,3-a]pyrimidin-5-one was prepared by Rashad et al., (2010). M.p. 250$252{ }^{\circ} \mathrm{C}$. IR spectrum $\left(\mathrm{KBr}, v, \mathrm{~cm}^{-1}\right): 3334$ $(\mathrm{NH}), 1643(\mathrm{CO}) ;{ }^{1} \mathrm{H}$ NMR spectrum $\left(\mathrm{CDCl}_{3}\right.$, $\delta \mathrm{ppm}): 3.36\left(\mathrm{~s}, 2 \mathrm{H}, \mathrm{CH}_{2}\right), 6.90\left(\mathrm{~s}, 1 \mathrm{H}, \mathrm{C}_{3}-\mathrm{H}\right)$, 7.24-7.54 (m, 4H, Ar-H), $9.85(\mathrm{~s}, 1 \mathrm{H}, \mathrm{NH}$, $\mathrm{D}_{2} \mathrm{O}$ exchangeable); ${ }^{13} \mathrm{C}$ NMR (DMSO- $\mathrm{d}_{6}, \delta$ ppm): 36.58 (C-10), 119.45-164.32 (Ar-C), $170.50(\mathrm{C}=\mathrm{O})$. MS, $m / z(\%): 280\left(\mathrm{M}^{+}, 100\right)$. Anal. calcd. for $\mathrm{C}_{14} \mathrm{H}_{8} \mathrm{~N}_{4} \mathrm{OS}$ : C, 59.99; H, 2.88; N, 19.99; S, 11.44. Found: C, 60.03; H, 2.95; N, 19.89; S, 11.38.

Compound number eight: 11-Amino-5,6dihydronaphtho $\left[1^{\prime}, 2^{\prime}: 4,5\right]$ thieno $[2,3-\mathrm{d}]$ pyrimidine- $9(8 \mathrm{H})$ - thione was prepared by Abdel-Megeid et al., (1998). M.p. $150-2^{\circ} \mathrm{C}$; Calcd. for $\mathrm{C}_{15} \mathrm{H}_{11} \mathrm{ClN}_{2} \mathrm{~S}$ (286.78): C, 63.04; $\mathrm{H}$, 3.85; N, 9.80; S, 11.20. Found: C, 63.80; H, $3.83 ; \mathrm{N}, 9.40 ; \mathrm{S}, 1120$. IR (KBr, $\left.v, \mathrm{~cm}^{-1}\right): 1055$ (C-Cl) ; ${ }^{1} \mathrm{H}$ NMR (DMSO-d $6, \delta$ ppm): 2.7 (s, $\left.3 \mathrm{H}, \mathrm{CH}_{3}\right), 2.8-3.0\left(\mathrm{~m}, 4 \mathrm{H}, \mathrm{CH}_{2} \mathrm{CH}_{2}\right), 7.2-7.4$ (m, 3H, Ar-H) ; MS, $m / z(\%): 288\left(\mathrm{M}^{+},{ }^{37} \mathrm{Cl}\right.$, 38.4), $286\left(\mathrm{M}^{+},{ }^{35} \mathrm{Cl}, 100\right), 271$ (1.47), 251 (7.25),249 (9.28), 209 (9.51),164 (7.65), 139 (4.86).

Compound number nine: 11-Hydrazino-9methyl-5,6-dihydronaphtho[ $\left[1^{\prime}, 2^{\prime}: 4,5\right]$ thieno [2,3-d]pyrimidine was prepared by AbdelMegeid et al., (1998). M.p. $182-4{ }^{\circ} \mathrm{C}$; IR ( $\mathrm{KBr}$, $\left.v, \mathrm{~cm}^{-1}\right): 3400-3440\left(\mathrm{NH}_{2}\right), 3200(\mathrm{NH}) ;{ }^{1} \mathrm{H}$ NMR $\left(\mathrm{CDCl}_{3}, \delta \mathrm{ppm}\right): 2.6\left(\mathrm{~s}, 3 \mathrm{H}, \mathrm{CH}_{3}\right), 2.8$ $3.0\left(\mathrm{~m}, 4 \mathrm{H}, \mathrm{CH}_{2} \mathrm{CH}_{2}\right), 4.2\left(\mathrm{~s}, 2 \mathrm{H}, \mathrm{NH}_{2}, \mathrm{D}_{2} \mathrm{O}\right.$ exchangeable $), \quad 6.45 \quad\left(\mathrm{~s}, \mathrm{H}, \quad \mathrm{NH}, \quad \mathrm{D}_{2} \mathrm{O}\right.$ exchangeable), 7.2-7.5 (m, 4H, Ar-H); MS, $m / z \quad(\%): 282 \quad\left(\mathrm{M}^{+}, 100\right), 264$ (38.24), 224 (5.89), 209 (6.62), 164 (8.22), 139 (8.9).
Compound number ten: 11-Chloro-9-methyl5,6- dihydronaphtho $\left[1^{\prime}, 2^{\prime}: 4,5\right]$ thieno [2,3-d] pyrimidine was prepared by Abdel-Megeid et al., (1998). M.p. $150-2^{\circ} \mathrm{C}$; Calcd. for $\mathrm{C}_{15} \mathrm{H}_{11} \mathrm{ClN}_{2} \mathrm{~S}$ (286.78): C, 63.04; H, 3.85; N, 9.80; S, 11.20. Found: C, 63.80; H, 3.83; N, 9.40; S, 1120. IR ( $\left.\mathrm{KBr}, v, \mathrm{~cm}^{-1}\right): 1055(\mathrm{C}-\mathrm{Cl})$; ${ }^{1} \mathrm{H}$ NMR (DMSO-d $\left.\mathrm{d}_{6}, \delta \mathrm{ppm}\right): 2.7\left(\mathrm{~s}, 3 \mathrm{H}, \mathrm{CH}_{3}\right)$, 2.8-3.0 (m, $\left.4 \mathrm{H}, \mathrm{CH}_{2} \mathrm{CH}_{2}\right)$, 7.2-7.4 (m, 3H, Ar$\mathrm{H})$; MS, $m / z(\%): 288\left(\mathrm{M}^{+},{ }^{37} \mathrm{Cl}, 38.4\right), 286$ $\left(\mathrm{M}^{+},{ }^{35} \mathrm{Cl}, 100\right), 271 \quad(1.47), 251 \quad(7.25), 249$ (9.28), 209 (9.51), 164 (7.65), 139 (4.86).

Compound number eleven: 5-Amino-1-(9methyl-5, 6- dihydronaphtho $\left[1^{\prime}, 2^{\prime}: 4,5\right]$ thieno $[2, \quad 3-d]$ pyrimidin-11-yl) -1H-pyrazole-4carboxylic acid methyl ester was prepared by Rashad et al., (2009). M.p. 139-141 ${ }^{\circ}$ C. IR v 3464, $3354\left(\mathrm{NH}_{2}\right), 1685(\mathrm{CO}) \mathrm{cm}^{-1} .{ }^{1} \mathrm{H}$ NMR $\left(\mathrm{DMSO}_{-} \mathrm{d}_{6}, \delta \mathrm{ppm}\right): 33(\mathrm{t}, \mathrm{J}=6.9 \mathrm{~Hz}, 3 \mathrm{H}$, $\left.\mathrm{CH}_{3}\right), 2.81\left(\mathrm{~s}, 3 \mathrm{H}, \mathrm{CH}_{3}\right), 2.95-3.07(\mathrm{~m}, 4 \mathrm{H}$, $\left.2 \mathrm{CH}_{2}\right), 4.28\left(\mathrm{q}, \mathrm{J}=7.5 \mathrm{~Hz}, 2 \mathrm{H}, \mathrm{CH}_{2}\right), 6.43(\mathrm{~d}, \mathrm{~J}$ $=8 \mathrm{~Hz}, 1 \mathrm{H}, \mathrm{Ar}-\mathrm{H}), 6.81\left(\mathrm{~s}, 2 \mathrm{H}, \mathrm{NH}_{2}, \mathrm{D}_{2} \mathrm{O}\right.$ exchangeable), $6.90-7.35(\mathrm{~m}, 4 \mathrm{H}, 3 \mathrm{Ar}-\mathrm{H}$ and $\left.\mathrm{C}_{3}-\mathrm{H}\right) .{ }^{13} \mathrm{C}$ NMR (DMSO-d $\mathrm{d}_{6}, \delta \mathrm{ppm}$ ): 14.4 $\left(\mathrm{CH}_{3}\right), 25.1\left(\mathrm{CH}_{3}\right), 25.2(\mathrm{C}-5), 29.3\left(\mathrm{C}^{\prime} 6^{\prime}\right)$, $59.8\left(\mathrm{OCH}_{2}\right), 96.4(\mathrm{C}-4), 125.6-164.3(\mathrm{Ar}-\mathrm{C})$, 172 (CO). MS, $m / z$ (\%): $405\left(\mathrm{M}^{+}, 100\right)$. Anal. calcd for $\mathrm{C}_{21} \mathrm{H}_{19} \mathrm{~N}_{5} \mathrm{O}_{2} \mathrm{~S}: \mathrm{C}, 62.20 ; \mathrm{H}, 4.72 ; \mathrm{N}$, 17.27; S, 7.91. Found: C, 62.30; H, 4.65; N, 17.33; S, 7.81 .

Compound number twelve: 5-Amino-1-(9methyl-5, 6-dihydronaphtho $\quad\left[1^{\prime}, 2^{\prime}: 4,5\right]$ thieno[2,3- $d$ ]pyrimidin-11-yl)-3-

methylsulfanyl- $1 H$-pyrazole-4-carbonitrile was prepared by Rashad et al., (2009). M.p. 124$126{ }^{\circ} \mathrm{C}$. IR v 3460, $3346\left(\mathrm{NH}_{2}\right), 2215(\mathrm{CN})$ $\mathrm{cm}^{-1} .{ }^{1} \mathrm{H}$ NMR (DMSO-d $\left.6, \delta \mathrm{ppm}\right): 1.58(\mathrm{~s}$, $\left.3 \mathrm{H}, \mathrm{SCH}_{3}\right), 2.80\left(\mathrm{~s}, 3 \mathrm{H}, \mathrm{CH}_{3}\right), 2.90-3.05(\mathrm{~m}$, $\left.4 \mathrm{H}, 2 \mathrm{CH}_{2}\right), 6.50(\mathrm{~d}, \mathrm{~J}=8 \mathrm{~Hz}, 1 \mathrm{H}, \mathrm{Ar}-\mathrm{H}), 6.60$ (s, $2 \mathrm{H}, \mathrm{NH}_{2}, \mathrm{D}_{2} \mathrm{O}$ exchangeable), 7.00-7.25 (m, $3 \mathrm{H}, \mathrm{Ar}-\mathrm{H}) .{ }^{13} \mathrm{C}$ NMR (DMSO-d 6 , $\delta$ ppm): 11.6 $\left(\mathrm{CH}_{3}\right), 25.1\left(\mathrm{CH}_{3}\right), 25.3\left(\mathrm{C}^{-} 5^{\circ}\right), 29.3\left(\mathrm{C}-6^{\prime}\right)$, 113.0 (C-4), $115.5(\mathrm{CN}), 126.0-172.3$ (Ar-C). MS, $m / z(\%)$ : $404.09\left(\mathrm{M}^{+}, 100\right)$. Anal. calcd for $\mathrm{C}_{20} \mathrm{H}_{16} \mathrm{~N}_{6} \mathrm{~S}_{2}: \mathrm{C}, 59.38 ; \mathrm{H}, 3.99 ; \mathrm{N}, 20.78 ; \mathrm{S}$, 15.85. Found: C, 59.29; H, 4.05; N, 20.71; S, 15.92.

Compound number thirteen: 1-(5, 6Dihydronaphtho $[10,20: 4,5]$ thieno $[2,3-d]$ pyrimidin-11- yl)-1H-pyrazolo $[3,4-d]$ pyrimidin-4(3H)-one was prepared by Rashad 
et al., (2009). M.p. $265-267^{\circ} \mathrm{C}$. IR (KBr) nmax: $\left.\mathrm{cm}^{-1}: 3200(\mathrm{NH}), 1674(\mathrm{C}] \mathrm{O}\right), 1587$ $(\mathrm{C}] \mathrm{N}) .{ }^{1} \mathrm{H}$ NMR (DMSO-d $6, \delta$ ppm): d: 2.90 $3.00\left(\mathrm{~m}, 4 \mathrm{H}, \mathrm{C}_{50}-\mathrm{CH}_{2} \mathrm{C}_{60}-\mathrm{CH}_{2}\right), 7.20-7.30(\mathrm{~m}$, $\left.4 \mathrm{H}, 3 \mathrm{Ar}-\mathrm{H} \mathrm{C} \mathrm{C}_{3}-\mathrm{H}\right), 7.40-7.50$ (m, 2H, Ar-H $\mathrm{NH}, \mathrm{D}_{2} \mathrm{O}$ exchangeable), $8.40\left(\mathrm{~s}, 1 \mathrm{H}, \mathrm{C}_{6}-\mathrm{H}\right)$, $9.20\left(\mathrm{~s}, 1 \mathrm{H}, \mathrm{C}_{9}-\mathrm{H}\right) \cdot{ }^{13} \mathrm{C}$ NMR (DMSO-d $\mathrm{d}_{6}, \delta$ ppm): d: 23.50 (C-50), 29.30 (C- 60), $119,122,125.20,126,126.50,126.90,132$, $138, \quad 140.30,158.40, \quad 167.10 \quad \mathrm{sp}^{2}$ carbon atoms), $165(\mathrm{C}] \mathrm{O}) . \mathrm{MS} \mathrm{m} / \mathrm{z}(\%): 372(\mathrm{M}$, 27\%), $344\left(\mathrm{M}^{+}, \mathrm{CO}, 10 \%\right), 316(16 \%), 292$ (42\%). Anal. calcd. for $\mathrm{C}_{19} \mathrm{H}_{12} \mathrm{~N}_{6} \mathrm{OS}(372.41)$ : C $61.28, \mathrm{H} 3.25, \mathrm{~N} 22.57, \mathrm{~S}$ 8.61. Found: C 61.05, H 3.37, N 22.41, S 8.57.

\section{2- Antiviral Bioassays}

Cells: Madin-Darby Canine Kidney (MDCK) cells were maintained at the Environmental Virology Laboratory at the National Research Center. Madin-Darby canine kidney (MDCK) cells were kindly provided by Dr. Richard Webby, St. Jude Children's Research Hospital, Department of Virology and Molecular Biology, USA, as a confluent sheet in $75 \mathrm{~cm}^{2}$ tissue culture flask. The cell lines was propagated till confluence for several passages, harvested in aliquots and stored in liquid nitrogen till being used. MDCK cells are recommended by WHO as the preferred cell line for culturing influenza viruses.

Virus: The highly pathogenic avian influenza virus (HPAI) H5N1 used in this study was isolated from the infected chickens in Egypt in 2006 (characterized at immunologic and molecular

levels)

A/Chicken/Qalubiya/1/2006(H5N1) and was kindly provided by Prof. Dr. M. A. Ali professor of virology, National Research Center. All viral manipulations were performed under the appropriate biosafety level 3 laminar air flow isolator at Virology Lab., Department of water pollution, NRC.

Cell culture propagation: The MDCK cells were grown to confluence in complete growth medium in $75 \mathrm{~cm}^{2}$ culture flasks (Greiner bioone $\mathrm{GmbH}$, Germany). The medium was discarded and then cells were treated with trypsin-versene for dissociation of the cell monolayer. Cells were incubated for 0.5-5 min at $37^{\circ} \mathrm{C}$ and detachment of the cells was carefully observed. Cells were spun down at $2500 \mathrm{~g}$ for $2 \mathrm{~min}$, resuspended in $1 \mathrm{ml}$ complete medium. The exact cell count and viability was recorded by mixing a $50 \mu \mathrm{l}$ aliquot of the cells with equal volume of trypan blue $(5 \mathrm{gm} / \mathrm{L}$; BiochromKG, Berlin, Germany) using a hemocytometer (Right Line; Sigma, Deisenhofen, Germany). According to the recorded count certain volume of the resuspended cells was used to prepare fresh cultures in plates or flasks.

Viral titer determination by plaque assay: Monolayer MDCK cells $\left(6 \times 10^{5}\right.$ cells/well $)$ were washed once with Dulbecco's phosphatebuffered saline (DPBS), and infected with a serially diluted viral suspension. After adsorption for $1 \mathrm{~h}$ at $37^{\circ} \mathrm{C}$, the viral suspension was replaced with over layer medium, (DMEM with penicillin $[100 \mathrm{U} / \mathrm{mL}]$, streptomycin [100 $\mu \mathrm{g} / \mathrm{mL}]$, L-glutamine $[2 \mathrm{mM}$ ], and nonessential amino acid mixture $[0.1 \mathrm{mM}]$ ) containing 2.5 $\mu \mathrm{g} / \mathrm{mL}$ trypsin and $0.3 \%$ agarose). After incubation for $2-3$ days at $37^{\circ} \mathrm{C}$ under $5 \%$ $\mathrm{CO}_{2}$, the cells were fixed with $10 \%$ formaldehyde and then stained with $1 \%$ crystal violet. The titer of the virus was expressed in plaque-forming units (PFU) per milliliter.

Preparation of synthetic compounds for bioassay: Tested compounds were dissolved as $10 \mathrm{mg}$ each in $1 \mathrm{ml}$ of $10 \%$ Dimethyl Sulfoxide (DMSO) in deionized water $(900 \mu \mathrm{L}$ de-ionized water and $100 \mu \mathrm{L}$ DMSO). Decontamination was carried out by adding 1 $\%$ antibiotic-antimycotic mixture $(10,000 \mathrm{U}$ penicillin $\mathrm{G}$ sodium, $10,000 \mu \mathrm{g}$ streptomycin sulfate, and $250 \mu \mathrm{g}$ amphotericin B, PAA Laboratories $\mathrm{GmbH}$, Austria) and the compounds were incubated at $37^{\circ} \mathrm{C}$ for $30 \mathrm{~min}$ then stored at $-20^{\circ} \mathrm{C}$. Sterility test was performed out in nutrient agar to ensure the sterility of the prepared compounds.

Cytotoxicity Assay: Cell toxicity was carried out to determine the cell culture safe doses of the dissolved compounds and performed by Cell morphology technique (Aquino et al., 1989) which was made in 96 well plates: In order to determine the safe dose of the tested compounds that doesn't harm the (MDCK) cells, different concentrations from each compound ranging from 10 to $100 \mu \mathrm{g} / \mathrm{ml}$ 
were applied to cells. Each concentration was tested for cytotoxicity by observing any morphologically changes in the cultured cells microscopically after 24 hours incubation.

Antiviral Screening: Plaque reduction assay is the most widely accepted method for determining the \% inhibition of virus as a result of being subjected to a given material (Tebas et al., 1995). A 12 well plate was cultivated with the MDCK cells $\left(10^{5} \mathrm{cell} / \mathrm{ml}\right)$ and incubated for 24 hours at $37^{\circ} \mathrm{C}$. Virus was diluted to give $10^{3} \mathrm{PFU} / \mathrm{ml}$ final concentration and mixed with the safe concentrations of each compound as mentioned previously and incubated for 1 hour at $37^{\circ} \mathrm{C}$. Growth medium was removed from the multi-well plate and virus-compound mixture was inoculated in each well. After 1 hour contact time at $37^{\circ} \mathrm{C}$ for virus adsorption, the inoculum was aspirated and $1 \mathrm{ml}$ of cell-specific $2 \mathrm{x}$ medium $2 \%$ agarose was overlayed the cell sheet. The plates were left to solidify at room temperature and incubated for $2-3$ days at $37^{\circ} \mathrm{C}$ until the development of the viral plaques. Formalin was added for two hours for fixation then plates were stained with crystal violet staining solution. Control virus and cells were treated identically without addition of compounds. Viral plaques were counted and the percentage of virus reduction was calculated through the following equation: $\%$ inhibition $=$ viral count (untreated) - viral count (treated)/ viral count (untreated) x 100 .

\section{RESULTS AND DISCUSSION}

The result in Fig. 1 showed that the dilution $10^{-3}$ of $\mathrm{H} 5 \mathrm{~N} 1$ virus was the most suitable dilution which gave $80-100$ $\mathrm{PFU} / \mathrm{ml}$. So, this dilution $\left(10^{-3}\right.$ of virus $)$ will be applied in subsequent experiments. Also the results of cytotoxicity test indicated that all tested concentrations starting from 10 to $100 \mu \mathrm{g} / \mathrm{ml}$ for each compound were safe on MDCK cells and didn't show any toxicity on MDCK cells.

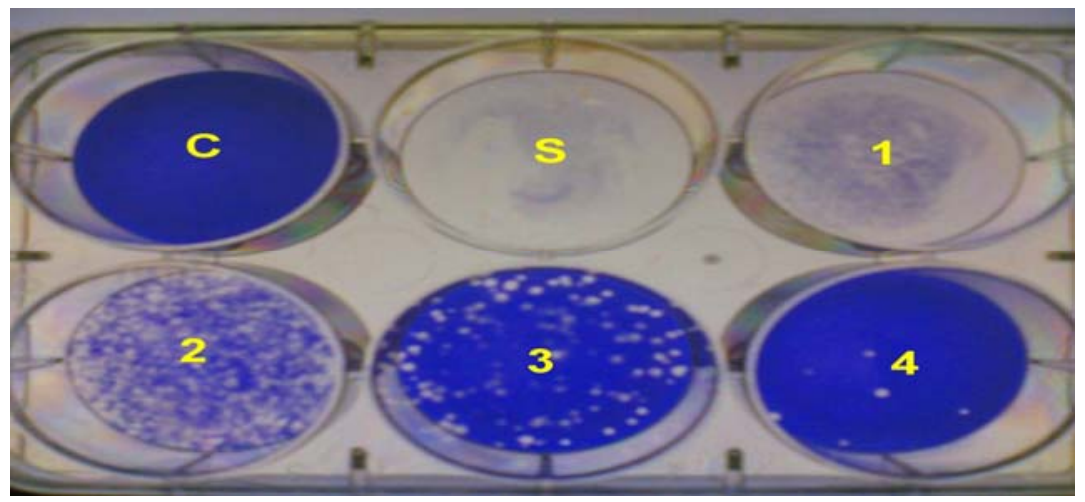

Fig. A: Plaque infectivity titration of $\mathrm{H} 5 \mathrm{~N} 1$ virus on $\mathrm{MDCK}$ cells $\mathrm{c}=$ cell control; $\mathrm{s}=$ stock virus (undiluted); 1, 2, 3, $4=$ serial dilutions of the virus stock $\left(10^{-1}, 10^{-2}, 10^{-3}, 10^{-4}\right)$ respectively used in treating each well. The fig. showed that the best dilution of virus used that gives (80-100) plaque $\mathrm{PFU} / \mathrm{ml}$ was $10^{-3}$.

The lowest concentrations $10 \& 20$ $\mu \mathrm{g} / \mathrm{ml}$ for each compound was preferably chosen to be used in plaque reduction assay since the commercially available anti-H5N1 therapeutic agents are effective at low concentration. This result agreed with several searches in that field for example, toxicity test using neutral red dye uptake method (Smee et. al, 2002), using $0.011 \%$ final concentration of the dye for $2 \mathrm{~h}$ for the compound 4-[(1,2-dihydro-2oxo-3H-indol-3-ylidene) amino]-N-(4,6dimethyl-2-pyrimidin-2-yl)benzenesulphonamide and its derivatives showed that no toxicity was apparent to the uninfected stationary MDCK cell monolayers at the 
highest $(100 \mu \mathrm{g} / \mathrm{ml})$ concentration (Selvam et al., 2006). A series of pyrazinecarboxamide derivatives were found to inhibits the influenza viral RNAdependent RNA polymerase without cytotoxicity to mammalian cells (Furuta et. al, 2009). The cytotoxicity test using MTT assay (Mossman, 1983) for some novel fused thiophene and thienopyrimidine derivatives showed that they were safe on MDCK cells (Rashad et. al, 2010). However, the acyclic nucleosides in the 5alkynyl- and 6-alkylfuro [2, 3-d] pyrimidine series showed cellular toxicity against PBM, CEM and VERO cells, probably by inhibiting cell DNA synthesis (Amblard et. al, 2005).

Plaque reduction assay was applied to get the percentage of virus inhibition as a result of treatment with certain compound. The results in Table 1 showed that 10 out of 13 compounds showed moderate percentage of inhibition at concentration $20 \mu \mathrm{g} / \mathrm{ml}$. These compounds were number $10,13,9,12,1,11,7,8,3$ and 4 giving $66.6,66.6,60.0,60.0,58.3$, $55.0,53.3,53.3,50.0$ and $50.0 \%$ of inhibition, respectively. The remaining 3 compounds showed weak inhibitory activity at same concentration. These compounds were number 5, 2 and 6 giving $35,33.3$ and $33.3 \%$ of inhibition, respectively. Generally, at conc. $10 \mu \mathrm{g} / \mathrm{ml}$ the percentage inhibition was slightly reduced for most tested compounds comparing with $20 \mu \mathrm{g} / \mathrm{ml}$ but this reduction was non significance.

Table 1: Inhibitory activity of synthetic compounds using plaque reduction count assay against Avian $\mathrm{H} 5 \mathrm{~N} 1$ virus.

\begin{tabular}{|c|c|c|c|c|}
\hline \multirow[b]{2}{*}{ Compounds code } & \multirow{2}{*}{$\begin{array}{l}\text { Conc. } \\
(\mu \mathrm{g} / \mathrm{ml})\end{array}$} & \multicolumn{3}{|c|}{ Antiviral effect (using MDCK). H5N1 } \\
\hline & & $\begin{array}{l}\text { Initial viral } \\
\text { count }\left(\times 10^{4}\right)\end{array}$ & $\begin{array}{c}\text { Viral count after } \\
\text { treatment } \\
(\mathrm{PFU} / \mathrm{ml})\left(\mathbf{x 1 0}^{4}\right) \\
\end{array}$ & $\begin{array}{c}\% \text { of } \\
\text { inhibition }\end{array}$ \\
\hline \multirow{2}{*}{1} & 10 & \multirow{4}{*}{12} & 5.4 & 55.0 \\
\hline & 20 & & 5.0 & 58.3 \\
\hline \multirow{2}{*}{2} & 10 & & 10 & 16.6 \\
\hline & 20 & & 0.8 & 33.3 \\
\hline \multirow{2}{*}{3} & 10 & \multirow{22}{*}{16} & 8.6 & 46.3 \\
\hline & 20 & & 8.0 & 50.0 \\
\hline \multirow{2}{*}{4} & 10 & & 9.2 & 42.5 \\
\hline & 20 & & 8.0 & 50.0 \\
\hline \multirow{2}{*}{5} & 10 & & 10.8 & 32.50 \\
\hline & 20 & & 10.4 & 35.0 \\
\hline \multirow{2}{*}{6} & 10 & & 8.6 & 28.3 \\
\hline & 20 & & 8.0 & 33.3 \\
\hline \multirow{2}{*}{7} & 10 & & 8.0 & 33.3 \\
\hline & 20 & & 5.6 & 53.3 \\
\hline \multirow{2}{*}{8} & 10 & & 10 & 16.6 \\
\hline & 20 & & 5.6 & 53.3 \\
\hline \multirow{2}{*}{9} & 10 & & 5.4 & 55.0 \\
\hline & 20 & & 4.8 & 60.0 \\
\hline \multirow{2}{*}{10} & 10 & & 5.0 & 58.3 \\
\hline & 20 & & 4.0 & 66.6 \\
\hline \multirow{2}{*}{11} & 10 & & 5.6 & 53.3 \\
\hline & 20 & & 5.4 & 55.0 \\
\hline \multirow{2}{*}{12} & 10 & & 8.6 & 28.3 \\
\hline & 20 & & 4.8 & 60.0 \\
\hline \multirow{2}{*}{13} & 10 & & 5.6 & 53.3 \\
\hline & 20 & & 4.0 & 66.6 \\
\hline
\end{tabular}


Several reports also have been demonstrated the effectiveness of synthetic fused heterocyclic pyrimidine compounds in inhibiting influenza viruses. For examples, The 4-[(1,2-dihydro-2-oxo- $3 \mathrm{H}-$ indol-3-ylidene) amino]- $N$-(4,6-dimethyl2-pyrimidin-2-yl) benzenesulphonamide and its derivatives showed antiviral potencies $\quad(90 \%$ inhibition $)$ against influenza A (H1N1, H3N2, and H5N1) and $\mathrm{B}$ viruses in MDCK cell culture (Selvam et al., 2006). The novel 4-(4-((3-(2-amino-4hydroxy-6-methyl-5-pyrimidinyl) propyl) amino) phenyl)-1-chloro-3-buten-2-one showed the ability to inhibit the avian influenza H5N1 virus (lee et al., 2009). Some derivatives containing dihydronaphtho, naphtho $[2,1-b]$ thiophene and thieno [2, 3-d] pyrimidine ring systems showed promising antiviral activity against $\mathrm{H} 5 \mathrm{~N} 1$ virus (Rashad et al., 2010). Pyrazole compound BPR1P0034 showed potent and selective anti-influenza virus activity (Shih et al., 2010). However, some newly synthesized indenothienopyrimidine derivatives didn't show any antiviral activity against $\mathrm{H} 5 \mathrm{~N} 1$ virus (Shamrokh et al., 2010) also the novel 4-Amino-2- (benzylthio) -6(4-methoxyphenyl) pyrimidine -5carbonitrile didn't possess any antiviral activity against $\mathrm{H} 5 \mathrm{~N} 1$ virus (Mahmoud et al., 2011) while the antiviral activity of the novel acyclic nucleosides in the 5-alkynyland 6-alkylfuro [2, 3-d] pyrimidine series against $\mathrm{H} 5 \mathrm{~N} 1$ virus were secondary to their toxic effects on MDCK cell culture (Amblard et al., 2005) .

\section{REFERENCES}

Abdel-Megeid, M. E.; Hassan, N.A.; Zahran, M. A. and Rashad, A.E. (1998). Sulfur letters, 21 (6):269-284.

Amblard, F.; Vincent, A.; Pierre, G.;

Raymond, F. and Luigi, A. (2005): Synthesis and antiviral activity of novel acyclic nucleosides in the 5alkynyl- and 6-alkylfuro [2, 3-d] pyrimidine series. Bioorganic \& Medicinal Chemistry 13: 1239-1248.

Aquino, R.; DE Simone, C. P.; Conti, C. and Stein, M.L. (1989): Plant metabolites; structure and in vitro antiviral activity of Quinovic acid glycosides from Uncaria Tomentosa and Guettarda platypoda, journal of natural products, 52(4):679-685.

Basler, C.F. and Aguilar, P.V. (2008): Progress in identifying virulence determinants of the $1918 \mathrm{H} 1 \mathrm{~N} 1$ and the Southeast Asian H5N1 influenza A viruses. Antiviral Research 79: 166-178.

Cristescu, C. and Czobor, F. (1998): Astriazine derivatives with potential therapeutic action. XXVI. Synthesis of some 5-substituted-6-azauracil acyclonucleosides. Nucleos. Nucleot., 17: 1319-1324.

Furuta, Y.; Takahashi, K.; Shiraki, K.; Sakamoto, K. and Morrey, J. (2009): T-705 (favipiravir) and related compounds: Novel broad-spectrum inhibitors of RNA viral infections. Antiviral Research 82: 95-102.

Gong, J.; Xu, W. and Zhang, J. (2007): Structure and Functions of Influenza Virus Neuraminidase. Current Medicinal Chemistry, 14: 113-122.

Gubareva, L.V.; Matrosovich, M.N.; Brenner, M.K.; Bethell, R.C. and Webster, R.G. (1998): Evidence for zanamivir resistance in an immunocompromised child infected with influenza B virus. J. Infect. Dis., 178: 1257-1262.

Hegab, M. I.; Hassan, N. A.; Rashad, A. E.; Fahmy, A. A. and Abdel-Megeid, M. E. (2007). Phosphorus, Sulfur, and Silicon, 182:1535-1556.

Hsieh, H.P. and Hsu, J.T. (2007): Strategies of development of antiviral 
agents directed against influenza virus replication. Current pharmaceutical design, 13(34):3531-3542.

Kiso, M.; Mitamura, K.; Sakai-Tagawa,Y.; Shiraishi, K.; Kawakami, C.; Kimura, K.; Hayden, F.G.; Sugaya, N. and Kawaoka, Y. (2004): Resistant influenza A viruses in children treated with oseltamivir: descriptive study. Lancet 364: 759-765.

Le, Q.M.; Kiso, M.; Someya, K.; Sakai,Y.T.; Nguyen,T.H.; Nguyen, K.H.; Pham, N.D.; Ngyen, H.H.; Yamada, S.; Muramoto, Y.; Horimoto, T.; Takada, A.; Goto, H.; Suzuki, T.; Suzuki, Y. and Kawaoka, Y. (2005): Avian flu: isolation of drug-resistant H5N1 virus. Nature, 437: 1108.

Lee, D.C.; An, J.; Law, A.H.; Yang, C.L.; Poon, L.L.; Lau, A.S. and Jones, S.J. (2009): A novel small-molecule inhibitor of the avian influenza H5N1 virus determined through computational screening against the neuraminidase. J Med Chem. 2009 May 14; 52(9):2667-72.

Mahmoud Refaee Mahmoud; Ahmed Kamel El-Ziaty; Mahmoud Fawzy Ismail and Sayed Ahmed Shiba (2011): Synthesis of novel pyrimidine and fused pyrimidine derivatives. European Journal of Chemistry, 2(3): 347-355.

Mossman, T. (1983): J. Immunol. Methods 65, 55-63.

Rashad, A.E.; Heikal, O.A.; El-Nezhawy, A.O.H. and Abdel-Megeid, M.E. (2005). Heteroatom Chem. 16:226-234.

Rashad, A.E. and Ali, M.A. (2006): Synthesis and antiviral screening of some thieno[2,3-d] pyrimidine nucleosides. Nucleosides, Nucleotides, and Nucleic Acids, 25:17-28.

Rashad, A.E.; Hegab, M.I.; Abdel-Megeid, R.E.; Fathalla, N. and Abdel-Megeid, M.E. (2009): Synthesis and anti-HSV-1 evaluation of some pyrazoles and fused pyrazolopyrimidines. European Journal of Medicinal Chemistry 44:3285-3292.

Rashad, A.E.; Shamroukh, A.H.; AbdelMegeid, R.E.; Mostafa, A.; ElShesheny, R.; Kandeil, A.; Ali, M. A. and Banert, K. (2010): Synthesis and screening of some novel fused thiophene and thienopyrimidine derivatives for anti-avian influenza virus (H5N1) activity. European Journal of Medicinal Chemistry 45: 5251-5257.

Selvam, P.; Narayanan, M.; Markandavel, C.; Robert, W.; Wandersee, K. and Donald, F. (2006): Anti-influenza virus activities of 4-[(1,2-dihydro-2-oxo$3 \mathrm{H}$-indol-3-ylidene)amino]- $\mathrm{N}-(4,6-$ dimethyl-2-pyrimidin- 2yl)benzenesulphonamide and its derivatives. Antiviral Chemistry \& Chemotherapy 17:269-274.

Shamroukh, A. H.; Rashad, A. E. and Sayed, H. H. (2005). Phosphorus, Sulfur, and Silicon, 180:2347-2360.

Shamrokh, A. H.; Marzouk, N.A.; AlSaadny, A.H.; Micky, A. and AbdelMegeid, F. (2010): Anti-H5N1 Evaluation of some newly synthesized Indenothienopyrimidine derivatives. World Journal of Chemistry, 5(1): 37-43.

Shih, S.R.; Chu, T.Y.; Gadarla R.R.; Sung, N.T.; Hsiun, C.L.; Tang, W.F. and JimTong, H. (2010): pyrazole compound PR1P0034 with potent and selective anti-influenza virus activity. Journal of Biomedical Science, 17(13):1-9.

Smee, D.F.; Morrison, A.C.; Barnard, D.L. and Sidwell, R.W. (2002): Comparison of colorimeteric and visual methods for determining anti-influenza (H1N1 and H3N2) viruses activities and toxicities of compounds. Journal of Virological Methods 106:71-79.

Sugrue, R.; Tan, B.H.; Dawn, SY.Y. and Sutejo, R. (2008): Antiviral Drugs for 
the Control of Pandemic Influenza Virus. Annals Academy of Medicine, 37 (6):518-524.

Tapper, M.L. (2006): Emerging viral diseases and infectious disease risks. Hemophilia 12 (Suppl. 1): 3-7.
Tebas, P.; Stabell, E. C. and Olivo, P. D. (1995): Antiviral susceptibility testing with a cell line which expresses betagalactosidase after infection with Herpes Simplex Virus. Antimicrob. Agents Chemother., 39(6):1287- 1291.

\section{ARABIC SUMMARY}

فحص بعض المركبات المخلقة من المشتقات الحلقية للبيريمياينات كمضاد لفيروس أنفلونزا الطيور( إتش 5 إن 1)

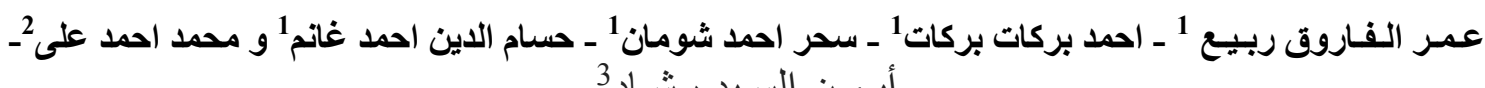

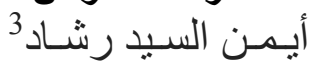

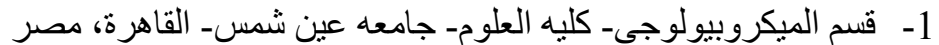

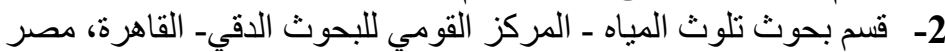

3- قسم الكيمياء الضوئية , المركز القومي للبحوث, الدقي , القاهرة, مصر الفرة

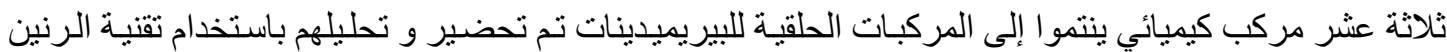

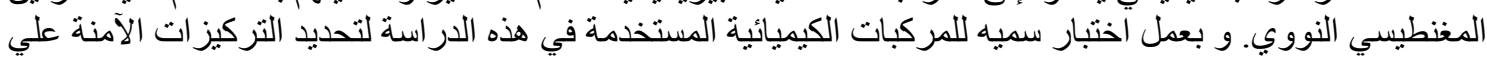

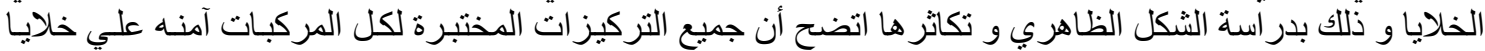

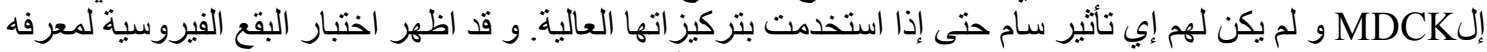

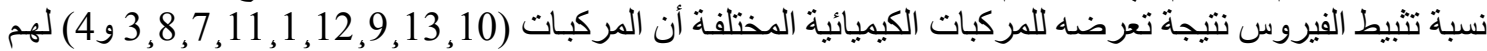

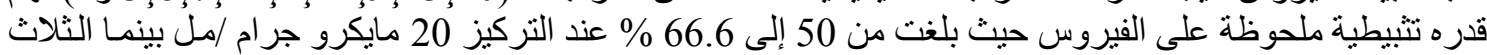
مركبات المتبقية (2,5 و 6 6) كانت لهم قدره تثبيطية ضعيفة على الفيروس عند نفس التركيز. 